\title{
Twins with Craniosynostosis: An Unidentified Variant/Twin Research: Kangaroo Care for Premature Twins; Developmental Delay in MZ Twins; Osteosarcoma in One Identical Twin; Controversies in Twin Pregnancy Management/Media Reports: Twin and Triplet Olympians; Twin's Rescue from a Crocodile; Twin Pandas in Japan; Twin Surrogacy; Identical Twins in Pennsylvania
}

\author{
Nancy L. Segal \\ Department of Psychology, California State University, Fullerton, CA, USA
}

\begin{abstract}
Craniosynostosis involves the early closure of one or more joints connecting the bones of an infant's skull. A case of young monozygotic (MZ) male twins with an unidentified variant of this condition is described, followed by a summary of relevant published reports. This overview is followed by descriptions of a kangaroo care program for premature twins, developmental delay in an MZ twin pair, osteosarcoma in one MZ twin and controversial issues in the management of multiple pregnancies. Media reports of twin and triplet Olympic athletes, a twin's rescue from a crocodile, the birth of twin pandas in Japan, a case of twin surrogacy and the birth of identical triplets are also presented.
\end{abstract}

\section{Twins with Craniosynostosis: An Unidentified Variant}

In July 2021, I received an email message from the father of an intriguing pair of young monozygotic (MZ) twin boys. His 8 -year-old sons had been born with a very rare condition known as craniosynostosis, albeit to different degrees of clinical severity. In brief, the key feature of craniosynostosis is the early coming together of the bones in a newborn's skull, prior to the full development of the brain. Typically, the bones begin to fuse over the first two years of life, although complete development occurs later. If craniosynostosis is left surgically uncorrected, the skull becomes further misshapen.

The twins' monozygosity was confirmed by DNA testing, and placental examination revealed two chorions and two amnions, an arrangement characterizing approximately one-third of MZ twin pairs (Segal, 2017a). Various forms of the condition are recognized, depending on where the early closures occur (Center for Disease Control, 2020). However, a DNA variant linked to the twins' specific form of sagittal craniosynostosis (in which bone fusion runs along the top of the head) has eluded physicians.

Author for correspondence: Nancy L. Segal, Email: nsegal@fullerton.edu Cite this article: Segal NL. (2021) Twins with Craniosynostosis: An Unidentified Variant/Twin Research: Kangaroo Care for Premature Twins; Developmental Delay in MZ Twins; Osteosarcoma in One Identical Twin; Controversies in Twin Pregnancy Management/Media Reports: Twin and Triplet Olympians; Twin's Rescue from a Crocodile; Twin Pandas in Japan; Twin Surrogacy; Identical Twins in Pennsylvania. Twin Research and Human Genetics 24: 352-357, https://doi.org/10.1017/thg.2021.39

\section{Frequency and Causes}

Overall, craniosynostosis affects approximately 1 in 2500 infants born in the USA. Sagittal craniosynostosis is, however, the most common form of the condition, comprising approximately $50 \%$ of the cases (Children's Health, 2021).

The causes of craniosynostosis are unknown, but appear to include the combined effects of genetic factors, such as mutations, and environmental factors, such as the fertility drug clomiphene (Center for Disease Control, 2020). In fact, 85\% of the cases are nonsyndromic and of unknown cause. However, several de novo mutations associated with craniosynostosis have been identified by exome sequencing of individuals with family member(s) who show the nonsyndromic midline form (Timberlake et al., 2017). Exome sequencing examines genes coding for proteins, that is, the functional portion of the genome (Eisenstadt, 2010), and is promising for detecting rare disease variants.

\section{The Causes}

I believe that the twins' case warrants close attention in the professional literature because it allows consideration of MZ cotwin differences in disease severity, within-pair differences in cotwin behavior and parental management of affected twin children. The family graciously offered to share their important story.

The twins were conceived 3 months after their mother had terminated birth control. Going off birth control may be a risk factor for multiple birth if conception occurs shortly thereafter (see Segal, 2000). The twins were delivered in February 2013 following an uneventful pregnancy that lasted for 35.5 weeks. 
Craniosynostosis was, however, diagnosed in one of the twins at the sixth or seventh month of pregnancy. Delivery was by cesarean section because of the condition of the newborn's skull; this twin was the second born. One or two days later, the same condition was diagnosed in the first born twin whom the doctors predicted would be unaffected. Both twins also had a septal atrial defect and oxygen around their lungs, a condition known as pneumothorax. Their birth weights and birth lengths were 5 pounds, 11 ounces and 20 inches, respectively (first born); and 5 pounds, 4 ounces and 18.20 inches. respectively (second born). These measures compare favorably with the mean birth weight (5 pounds, 4.45 ounces) and mean birth length (17.94 inches) of male twins born at 35 weeks to first-time mothers (Sankilampi et al., 2013). The twins' physical differences have been fairly constant across childhood, although at times their heights were the same. Both twins are right-handed.

\section{Parental Management}

The newborn twins remained in a high-level neonatal intensive care unit for 3 weeks prior to going home. Neither parent had a family history of craniosynostosis, although the mother noted that a distant cousin had cleft palate, which is one of several related conditions. The twins' parents were initially overwhelmed by the possible implications of their children's health, but learned that as long as craniosynostosis is not part of a larger syndrome, then the prognosis is favorable. However, managing the boys' early surgeries was challenging. At age 3 months, both twins underwent endoscopic strip craniectomy, a minimally invasive procedure that removes some bone from the area in which the early bone fusion occurred. A helmet can be worn to improve the shape of the infant's head (Ong, 2019). Unexpectedly, both twins' bones fused again almost immediately, a response that their surgeon had never observed during the course of his career. This outcome required cranial vault remodeling (reconstruction) when the twins were 10 months old - following removal of the bone, the head is reshaped and expanded (Children's Hospital of Philadelphia, 2021). Figure 1a shows one of the twins before and after early surgery. Figure $1 \mathrm{~b}$ shows them as young boys.

The twins' parents were understandably concerned about whether their twins' intellectual functions would be adversely affected. However, both twins have progressed well in their early years, achieving developmental milestones on schedule. Both twins are also doing Grade A work in school, but interestingly their work styles differ. One twin appears to master information quickly and easily, while his brother requires greater effort and concentration. Given these differences, their parents wisely placed them in different school classes. One twin is also more outgoing and gregarious, making decisions that his brother usually agrees to accept. Still, the twins are 'best friends' according to their parents — one twin insisted on sitting near the door of his classroom so he could keep his brother in view. I have observed that when twins are interacting freely with their peers in a playground setting, they may be apart physically, but stay attuned to the location of their sister or brother; this relationship feature is more characteristic of $\mathrm{MZ}$ than $\mathrm{DZ}$ twin pairs (Segal, 2017b). The twins have made many friends among the children in their neighborhood, evidence of their successful social skills.

Cranial surgeries such as those described above do not interfere with sports participation when patients are older. Both twins are currently active in athletics - their parents described their sons' physical feats as 'daring'. They have also observed that both boys dislike the anxiety associated with competitive team sports, preferring individual baseball and football activities.

The twins are doing well now medically, but will require further follow-up at age 10 years. They have already participated in a largescale study of de novo mutations possibly linked to craniosynostosis. Several mutations based on parent-child trios were implicated, with possible significance for other congenital conditions (Timberlake et al., 2017).

\section{Brief Research Review}

I was curious as to whether other studies of craniosynostosis were targeted to twins. A 2012 review identified 199 affected twins (Lakin et al., 2012) drawn from the medical literature and a small case series collected by the authors. Key findings were that (1) twinning was 2.62 times greater in patients $(6.29 \%)$ with craniosynostosis, compared with unaffected control participants (2.40\%); (2) more boys $(65.30 \%)$ than girls $(34.70 \%)$ were affected and (3) the MZ twin concordance rate $(60.90 \%)$ exceeded the DZ twin concordance rate $(5.30 \%)$. These findings support the role of both genetic and environmental factors in the origins of craniosynostosis.

The excess frequency of twinning reported in the 2012 review is particularly interesting. The twins' father has male cousins who are the twin children of his maternal aunt. He indicated that the twins were $\mathrm{DZ}$ and that one passed away 25 years ago from epilepsy that his cotwin did not have. In addition, the twins' maternal great aunt, maternal grandfather and maternal great-grandmother are lefthanded. The twins' father also has an older left-handed son from a previous marriage, but neither of the twins' parents nor the twins are left-handed.

Some researchers have found an association between twinning and left handedness (Derom et al., 1996; Suzuki \& Ando, 2014), although not all studies have concurred (Zheng et al., 2020). A study of handedness in families in which twins, as well as nontwins, were diagnosed with craniosynostosis might yield meaningful findings.

I located a 2017 case of MZ twins discordant for metopic craniosynostosis. Metopic craniosynostosis is a form of disorder in which the suture extends from the baby's nose to the sagittal suture at the top of the head (Center for Disease Control, 2020). This informative case sheds some light on the origins and progression of the condition (Magge et al., 2017).

An ultrasound taken at the 21st gestational week suggested a risk of trisomy 21 . The ultrasound also revealed monochorionic-diamniotic female twins, diagnosed as MZ based on later genetic testing. The only other concern was a unilateral choroid plexus cyst in one of the twins. (This cyst is a small fluid-filled structure, located in the lateral ventricles of the fetal brain. Its presence recommends examining the ultrasound for signs of aneuploidy, i.e. abnormal chromosome number; see Contemporary OB/GYN, 2013).

These twins were delivered vaginally to a primigravida at 32 weeks. Metopic craniosynostosis was identified in one of the twins when she was 2.5 months of age. In contrast, the condition was not diagnosed in her twin sister. Like the twin boys I described above, the affected twin underwent an endoscopic strip craniectomy and wore a cranial molding helmet for several months after the operation. Follow-up at age 4 years revealed no difference in cranial geometry between the two twins. Given the twins' discordance for metopic craniosynostosis, the researchers concluded that cranial suture fusion may involve a genetic predisposition that 
(a)

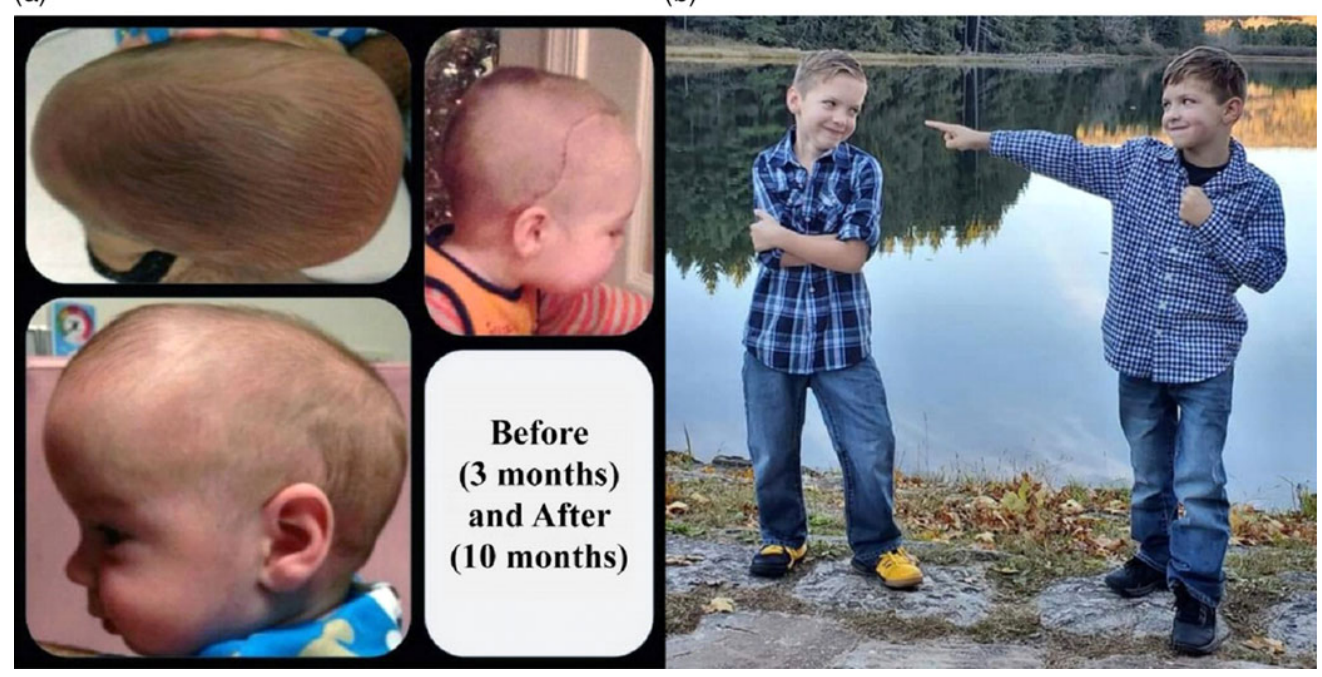

(b)

Fig. 1. A. One of the twins shown prior to having an endoscopic strip craniectomy (before 3 months) and following cranial vault remodeling (after 10 months). Courtesy of the twins' parents. B. The twins shown at 7 years of age playing in a park. Courtesy of the twins' sparents. requires activation by external factors and other epigenetic effects, such as intrauterine restraint. Continued study of such pairs promise to provide additional information on the origins, progression, management and possible prevention of craniosynostosis and other medical conditions.

\section{Twin Research}

\section{Kangaroo Care for Premature Twins}

Kangaroo care $(\mathrm{KC})$ is a practice that improves the health and wellbeing of both mother and newborn (Cleveland Clinic, 2020). Infant benefits include more rapid weight gain and increased sleeping time. The newborn baby, dressed only in a diaper, is held against the mother's bare chest for several hours each day; fathers can also administer KC. Maternal advantages include reduced anxiety and increased bonding. This practice is especially beneficial to babies who are premature and of low birth weight, both of which are relatively common among twins.

A program aimed to increase the practice of $\mathrm{KC}$ for twins born at tertiary care hospitals, known as the quality improvement (QI) project, was undertaken by researchers in Bengaluru, India (Arora et al., 2021). (Tertiary hospitals offer specialized treatment at a high level of care; Torrey, 2021). The collection of baseline data from April to July to 2019, and the training of hospital staff were completed to identify barriers (e.g. lack of patient privacy; shortage of nursing staff) to the successful implementation of $\mathrm{KC}$ in selected institutions. This prospective study included 169 twin pairs from among 238 twin deliveries. At the end of the study, the mean day of initiating $\mathrm{KC}$ shifted from day 8 after delivery to day 3 after delivery, and the duration of $\mathrm{KC}$ increased from $2.70 \mathrm{~h}$ per day to $7.88 \mathrm{~h}$ per day. The researchers recommended similar practices for use at other hospitals. Additional details about the twins, namely sex and zygosity, as well as details about cotwins with different developmental concerns, would have been informative with respect to the methods and outcomes described in this study.

\section{Developmental Delay in MZ Twins}

This interesting case study of 2-year-old MZ female twins was posed as a question to readers of the Journal of Paediatrics and Child Health, in a section titled 'Image of the Month' (Singanamalla et al., 2021). The image shows MZ twins with a triangular facial appearance, open mouth and broad root of the nose. As of 6 months of age, the twins displayed developmental delay and generalized tonic seizures that worsened with time. Other features included microcephaly, protruding tongue and abnormal brain waves that were recorded during sleep. The brain waves suggested hypsarrhythmia, a condition often associated with infantile spasms (Children's Hospital of Pittsburgh, n.d.). The answer to the diagnosis of the twin infants' condition was provided on a separate page.

The twins had Angelman syndrome (AS). As the authors explained, AS is a genetic disorder variously linked to a deletion of the $15 \mathrm{q} 11-\mathrm{q} 13$ region of maternal origin, mutations in the maternal UBE3A gene, an imprinting defect or paternal uniparental disomy. Unipaternal disomy involves inheriting two copies of a chromosome from one parent, rather than one copy from each parent. For example, AS can result when an infant receives two copies of a section of chromosome 15 from the father. In contrast, Prader-Willi syndrome results when an infant receives two copies of a section of chromosome 15 from the mother (Haldeman-Englert et al., 2021).

The method for establishing the twins' zygosity was not indicated by the investigators. Given the rarity of AS (1/10,000 to 1/ 24,000 births; Frohlich et al., 2019) and the twins' appearance, it is likely that they are MZ.

\section{Osteosarcoma in One MZ Twin}

A paper reporting three cases of $\mathrm{MZ}$ twin pair discordance for osteosarcoma (OS) is of interest. OS is the most common primary bone tumor detected in children and adults. However, it is rare in the population, occurring in 400-500 cases per year in the USA. Both the researchers and the twins are from China (Zhao et al., 2021). The three affected twins are all female and of ages 16 years (Case 1), 6 years (Case 2) and 20 years (Case 3 ). At the time of publication only, the affected 6-year-old twin was still living.

Case 1 was referred for medical assessment in 2017, due to her 3 -month period of knee pain. Case 2 came to attention in 2016, due to the twin's 2-month period of knee pain. Case 3 was referred in 2018, due the twin's knee pain associated with a fall. Cases 1 and 2 did not have histories that included trauma, infection, irradiation or cancer. Case 3 had no family history of malignant tumors or irradiation, but the patient had experienced trauma to the knee. All three of their cotwins remained healthy. In addition to these 
patients, Zhao et al. (2021) provided a summary table that includes clinical details for 42 cases of OS concordance in sibling sets that included one MZ twin pair. The sibling sets were variously composed of 2 siblings, 3 siblings and 4 siblings, for a total of 42 individuals. The combined studies included 25 males and 17 females with a mean age of 19 years and an age range spanning 3-57 years. This table should be especially helpful to investigators in the fields of genetics and oncology.

The research team concluded that while OS in MZ twins occurs rarely, such cases offer opportunities to assess the genetic factors and molecular mechanisms underlying this condition. Next-generation sequencing was recommended. However, a serious limitation of this study is that procedures for establishing the zygosity of the twins in the three cases were not provided. In the event that one or more of the affected cotwins was DZ, the interpretation and implications of this work would differ.

\section{Controversies in Twin Pregnancy Management}

A review of key controversies and challenges in the management of multiple birth pregnancies is an informative comprehensive guide for physicians, families and anyone concerned with the health and well-being of newborn infant twins and the parents of these multiples. The different areas and topics are listed below with a brief summary of selected issues.

Screening for aneuploidy. Noninvasive prenatal testing (NIPT) is common in singletons. However, NIPT has a higher failure rate in twin pregnancies, due to dichorionicity, in vitro fertilization, higher maternal weight, nulliparity and increased maternal age.

Assessment of fetal growth. Twins have different growth trajectories than nontwins. However, singleton growth charts are often used to assess their developmental progress.

Management of fetal growth restriction (FGR). Physicians differ as to the best criteria for identifying FGR. FGR increases twins' risks for perinatal mortality and morbidity, so it is important for hospital staff to be aware of the potential hazards.

Management of twin-to-twin transfusion syndrome (TTTS). The diagnostic criteria and treatment of early TTTS remain controversial. In addition, timing of delivery following laser treatment for TTTS is usually done at 34 weeks, but 37 weeks may be considered in the absence of additional pathology.

Management of twin anemia polycythemia sequence (TAPS). TAPS is a form of TTTS that complicates monochorionic twin pregnancies. Diagnostic procedures for TAPS remain an issue. There is a lack of consensus as to the best ways to manage TAPS because decisions rest on gestational age at diagnosis, disease severity, feasibility of intrauterine intervention and maternal preference.

Management of twin reversed arterial perfusion (TRAP) sequence. TRAP is a severe form of TTTS in monochorionic twins. The time of treatment has usually been 16 weeks, but recent data suggest that earlier treatment may be beneficial. Ultrasonic monitoring is recommended in cases of late diagnosis.

Management of Monochorionic-Monoamniotic (MCMA) twin pregnancy. The best antenatal management of MCMA twins has not been conclusively determined. Regular monitoring has been advocated by some physicians, while others believe it has limited value. Based on recent findings, it is suggested that MCMA twins be delivered between 32 and 33 weeks' gestation.

Screening and prevention of pre-eclampsia. Pre-eclampsia involves high maternal blood pressure and usually kidney and/or liver damage at the 20th gestational week (Mayo Clinic, 2020). The efficacy of the screening tool for pre-eclampsia (based on a combination of maternal risk factors, serum biochemistry, mean arterial pressure and uterine artery Doppler) has been based mostly on singleton pregnancies. More limited assessment in twin pregnancies suggests some difficulties. Prevention by aspirin prophylaxis has been used in some cases, but the optimal dosage remains at issue.

Management of single intrauterine demise (sIUD). Chorionicity affects sIUD, with monochorionic twins facing greater risk than dichorionic twins. The best time for delivery of the remaining twin is uncertain. It has been shown that earlier sIUD is associated with later gestational age at delivery.

Management of discordant anomalies. Discordant anomalies complicate $1 \%-2 \%$ of twins, with $80 \%$ of these cases including only one affected twin. Chorionic villus sampling can detect concordance or discordance when two placentas are available; however, misdiagnoses can occur when twins share a placenta. In addition, decisions surrounding selective termination of one twin are difficult in the event that a serious anomaly is discovered. Recent research showed no difference in outcomes between selective termination and expectant management in discordant dichorionic twins.

Screening, prevention and management of preterm birth. The best methods for preventing preterm delivery of twins and other multiples are under debate. In order to bring clarity to this question, there is a need for routine cervical-length assessment in the first trimester, in conjunction with the administration of vaginal progesterone to women with a short cervix. The optimal form of delivering twins is also uncertain, but it is recommended that cesarean section be offered to women laboring between 26 and 32 weeks with a non-cephalic presenting twin.

Timing of birth in uncomplicated twin pregnancy. Studies have produced mixed evidence as to whether the crown-to-rump length of the larger or smaller twin best reflects the length of the pregnancy. However, using the smaller twin's measure may miss knowing whether the growth of the twin is proceeding normally and may overlook possible aneuploidy. It is suggested that delivery of dichorionic twins occur at 37 weeks and delivery of monochorionic-diamniotic twins occur at 36-37 weeks.

Role of corticosteroids. The benefits of providing corticosteroids to women pregnant with twins and with nontwins have been debated. When given in nontwin pregnancies, corticosteroids may reduce the rates of perinatal death, respiratory distress syndrome, intraventricular, hemorrhage and necrotizing enterocolitis (intestinal inflammation in premature infants); however, there is a risk to the mother of maternal hyperglycemia. The benefits for twin versus nontwin pregnancies are currently undergoing study in a randomized controlled trial.

Mode of delivery. It is recognized that second-born twins are at higher physical risk than first-born twins. Current 
recommendations are to inform women that both cesarean section and vaginal delivery are safe if the pregnancy is uncomplicated, the presenting twin is cephalic and there are no marked twin size differences or other unusual factors that would need consideration.

\section{Media Reports}

\section{Twin and triplet olympians}

Both cotwins from eight pairs, single cotwins from two twin pairs, one individual male triplet and two female co-triplets were among the athletes competing at the Tokyo 2020-2021 Olympic games. The pairs included seven female sets and two male sets, all of whom appear to be identical based on photographs (Lakritz, 2021); one pair (the de Brower sisters) are actually two members of a triplet set. These multiples represented a variety of sports, including rhythmic gymnastics (Arina and Dina Averina-Russia), artistic gymnastics (Jennifer and Jessica Gadirova-Great Britain; Sanne and Lieke Wevers-the Netherlands; and Asia and Alice D'Amato-Italy), cycling (Adam and Simon Yates-Great Britain), boxing (Pat and Luke McCormack Great Britain) and synchronized swimming (Laura and Charlotte Tremble-France; Bregje and Noortje de Brouwer-the Netherlands and Anna-Maria and Eirini Alexandri-Austria); see Tokyo 2020 (2021).

Virtually every twin was happy to be part of the Olympics with their twin brother or sister. The twins competing in synchronized swimming would seem to have particular advantages in their sport, given that they compete as a team and their body movements would be coordinated naturally, due to their genetic identity. Former elite identical twins who took part in synchronized swimming are Karen and Sarah Josephson of the USA. Interestingly, a nontwin swimming team, Carolyn Waldo and Michelle Cameron, prepared to defeat the Josephson twins at the 1988 Olympics by purposefully matching their heartbeats and walking styles; however, these unrelated teammates could not do this successfully. In contrast, most identical twins' physical traits are coordinated without effort (see Segal, 2000). However, none of the twin and triplet pairs in synchronized swimming placed in the top three places that would have earned them medals (Tansey, 2021). Clearly, many performance factors affect the scores.

One of the individual twin competitors was Jake Gibb who is on the USA beach volleyball team (NBC News, 2021, July 27). He and his twin brother, Coleman, are the youngest of 11 children that include 6 boys and 5 girls (Celebrity Bio, 2021). I was unable to locate a picture of both twins; however, based on their birth order and mother's parity (and probable older age), it is likely that they are fraternal twins. However, these twins could not be entered into a scientific study due to their uncertain zygosity. The other individual twin competitor is track and field athlete Gabrielle Thomas who has a twin brother named Andrew. Thomas won a bronze medal in the $200-\mathrm{m}$ race, having placed third (CBS News, 2021).

The individual Olympic triplet was Jay Litherland who is a highly accomplished swimmer (NBC News, 2021, July 26). His triplet brothers, Kevin and Mick, are also swimmers; all three young men have been part of the University of Georgia's swim team. The brothers were born in Osaka to a mother from Japan and a father from New Zealand. Two of the three triplets, but possibly the third one as well, appear to be identical from their photographs. Litherland won a silver medal in the men's $400-\mathrm{m}$ individual swimming medley (Samson, 2021).

It is likely that other individual 'hidden' twins are participating in the Tokyo Olympics. It would be of interest to know their zygosity and why only one twin or triplet competed.

\section{Twin's Rescue from a Crocodile}

Melissa Laurie, a 28-year-old British twin, was attacked by a crocodile when she and her sister were swimming near Puerto Escondido in Mexico (BBC News, 2021). She was saved by her sister Georgia who punched the crocodile repeatedly. Melissa developed sepsis from her wounds and was put into an induced coma for recovery. She is now out of the coma, able to breathe on her own and appears to be aware of her surroundings. She is unable to speak because of soreness caused by the breathing tube. The twins had visited Mexico to work in animal sanctuaries and to travel. Challenging a crocodile is not easy, but it had to be done to save the life of a twin sister.

\section{Twin Pandas in Japan}

After 4 years of sexual abstinence, a 15-year-old male panda named $\mathrm{Ri} \mathrm{Ri}$ initiated a mating ritual with his partner Shin Shin (Hida, 2021). The pandas reside at the Ueno Zoo in Tokyo, Japan. In early June, the zoo staff announced that Shin Shin was pregnant and shortly thereafter they were delighted to witness the birth of twins, delivered an hour and a half apart. A zoo spokesman noted that panda mothers typically breastfeed only one twin, jeopardizing the well-being and survival of the other twin. Therefore, they arranged to keep the other twin in an incubator while their mother fed the twin, before having them exchange places.

\section{Twin Surrogacy}

Identical twins, Amy Fuggiti and Courtney Essenpreis, from Chicago, both suffer from Axenfeld-Rieger syndrome (Stern, 2021). This autosomal dominant condition has a $50 \%$ chance of being transmitted from parent to child. It mostly affects the eyes, but may also affect the teeth and facial bones (National Institute of Health, 2021). In an effort to prevent transmission of the disease to a child, Courtney conceived two healthy babies via in vitro fertilization (IVF). In contrast, Amy's attempts at becoming pregnant were unsuccessful. Apparently without discussion, the twins understood that Courtney would be implanted with a zygote created by her sister and brother-in-law. As her quote in the title says: 'It was the natural progression.' Courtney received a due date in October 2021.

\section{Identical Triplet Birth}

Identical female triplets, Anastasia, Olivia and Nadia, were born on July 8, 2021 at the University of Pennsylvania Hospital (Vitarelli, 2021). They had a shared placenta and separate amniotic sacs. The three infants are physically hard to distinguish, so their parents have applied different colored nail polish to the toes of two of their daughters and no coloring to the third. The pregnancy lasted for 34 weeks, with each newborn triplet weighing over 5 pounds. The triplets' gestational period and their birth weights compare favorably with those from a large study of Dutch twins (Lamb et al., 2011). Three triplet cohorts were assessed in that study; triplets born between 2000 and 2006; triplets born between 2000 and 2006 who survived for 28 days; and triplets enrolled in the Netherlands Twin Registry. The mean birth weights (in pounds) and pregnancy duration (in weeks), respectively, were 3.85 pounds, 31.60 weeks; 4.23 pounds, 31.70 weeks and 3.31 pounds, 30.8 weeks.

The triplets have two older sisters who appear to be excited to care for them. However, their enthusiasm may wane over the coming weeks and months as visitors will be intrigued by the triplets 
who have the dual status of being identical multiples and the babies of the family. It is important that their mother and father encourage others to pay attention to the two older girls who are just 3 and a half and 2 years of age. I have witnessed older siblings being overlooked by others when young multiple birth infants join the family. Staying aware of this possibility and planning ahead are paramount.

Acknowledgments. I am grateful to the parents of the twins I interviewed for the first part of this article. Their knowledge and reflections will assist other families in similar circumstances.

\section{References}

Arora, P., Kommalur, A., Devadas, S., Kariyappa, M., \& Rao, S. P. (2021). Quality improvement initiative to improve the duration of kangaroo mother care for twin preterm neonates born at a tertiary care hospital in resource-limited settings. Journal of Paediatrics and Child Health, 57, $1082-1088$.

BBC News. (2021, June 10). Crocodile attack: Twin Melissa Laurie is out of her coma. https://www.bbc.com/news/newsbeat-57430431

CBS News. (2021, August 3). Harvard grad Gabby Thomas wins bronze in women's 200-meter final in Tokyo. https://www.cbsnews.com/news/gabbythomas-womens-200-meter-bronze-medal-olympics/

Celebrity Bio. (2021). Jake Gibb. https://www.thecelebritybio.com/jake-gibb/ \#: :text=\%20Jake\%20Gibb\%20\%201\%20Jake\%20Gibb\%20has,tour\%20in\% 202000\%20alongside\%20Mike\%20Daniel.\%20More\%20

Center for Disease Control. (2020, October 23). Facts about craniosynstosis. https://www.cdc.gov/ncbddd/birthdefects/craniosynostosis.html

Children's Health. (2021). Pediatric sagittal craniosynostosis (scaphocephaly). https://www.childrens.com/specialties-services/conditions/sagittalsynostosis

Children's Hospital of Philadelphia. (2021). Crainosynostosis surgery. https:// www.chop.edu/treatments/surgical-treatment-craniosynostosis\#: $\sim:$ text $=$ Cranial\%20vault\%20remodeling\%2C\%20commonly\%20called\%20cranial\% 20vault\%20reconstruction $\% 2$ C,when\% 20 more\%20than $\% 20$ one $\% 20$ cranial\% 20suture\%20closes\%20prematurely.

Children's Hospital of Pittsburgh. (n.d.). What are infantile spasms? https:// www.chp.edu/our-services/brain/neurology/epilepsy/types/syndromes/ infantile-spasms

Cleveland Clinic. (2020, June 29). Kangaroo care. https://my.clevelandclinic. org/health/treatments/12578-kangaroo-care

Contemporary OB/GYN. (2013, April 1). Isolated fetal choroid plexus cysts. https://www.contemporaryobgyn.net/view/isolated-fetal-choroid-plexus-cysts

Derom, C., Thiery, E., Vlietinck, R., Loos, R., \& Derom, R. (1996). Handedness in twins according to zygosity and chorion type: A preliminary report. Behavior Genetics, 26, 407-408.

Eisenstadt, L. (2010, October 10). What is exome sequencing? https://www. broadinstitute.org/blog/what-exome-sequencing

Frohlich, J., Miller, M. T., Bird, L. M., Garces, P., Purtell, H., Hoener, M. C., Philpot, B. D., Sidorov, M. S., Tan, W. H., Hernandez, M. C., Rotenberg, A., Jeste, S. S., Krishnan, M., Khwaja, O., \& Hipp, J. F. (2019). Electrophysiological phenotype in Angelman syndrome differs between genotypes. Biological Psychiatry, 85, 752-759.

Haldeman-Englert, C., Freeborn, D., \& Turley, R. K. (2021). Uniparental disomy: Prader-Willi syndrome, Angelman syndrome. https://www.urmc. rochester.edu/encyclopedia/content.aspx?ContentTypeID $=90 \&$ ContentID $=$ P02159

Hida, H. (2021, June 23). A joyful surprise at Japan's oldest zoo: The birth of twin pandas. New York Times. https://www.nytimes.com/2021/06/23/world/ asia/tokyo-zoo-panda-twins.html

Lakin, G. E., Sinkin, J. C., Chen, R., Koltz, P. F., \& Girotto, J. A. (2012). Genetic and epigenetic influences of twins on the pathogenesis of craniosynostosis: A meta-analysis. Plastic and Reconstructive Surgery, 129, 945-954.
Lakritz, T. (2021, July 20). These 7 sets of twins are competing together at the Tokyo Olympics. Insider. https://www.insider.com/olympics-twinscompeting-together-2021-7\#twins-dina-and-arina-averina-of-russia-willboth-compete-in-rhythmic-gymnastics- 6

Lamb, D. J., Middeldorp, C. M., van Beijsterveldt, C. E., Vink, J. M., Haak, M. C., \& Boomsma, D. I. (2011). Birth weight in a large series of triplets. BMC Pediatrics, 11, 1-11.

Magge, S. N., Snyder, K., Sajja, A., DeFreitas, T. A., Hofherr, S. E., Broth, R. E., Keating, R. F., \& Rogers, G. F. (2017). Identical twins discordant for metopic craniosynostosis: Evidence of epigenetic influences. Journal of Craniofacial Surgery, 28, 14-16.

Mayo Clinic. (2020, March 19). Preeclampsia. https://www.mayoclinic.org/ diseases-conditions/preeclampsia/symptoms-causes/syc-20355745

National Institute of Health. (2021). Axenfeld-Rieger syndrome. https:// rarediseases.info.nih.gov/diseases/5701/axenfeld-rieger-syndrome

Ong, J. (2019, November 15). What is endoscopic strip craniectomy? https:// www.julingong.com/endoscopic-strip-craniectomy/

Samson, C. (2021, July 26). Jay Litherland becomes first Asian American winner at Tokyo Olympics. https://news.yahoo.com/jay-litherland-becomes-firstasian-180252663.html?fr=yhssrp_catchall

Sankilampi, U., Hannila, M. L., Saari, A., Gissler, M., \& Dunkel, L. (2013). New population-based references for birth weight, length, and head circumference in singletons and twins from 23 to 43 gestation weeks. Annals of Medicine, 45, 446-454.

Segal, N. L. (2000). Entwined lives: Twins and what they tell us about human behavior. Plume.

Segal, N. L. (2017a). Twin mythconceptions: False beliefs, fables, and facts about twins. Elsevier.

Segal, N. L. (2017b). Ethological studies using twins: Doing what comes naturally. Human Ethology Bulletin, 32, 35-43.

Singanamalla, B., Bhagwat, C., Madaan, P., Saini, L., \& Srivastava, P. (2021). Monozygotic twins with development delay and a characteristic electroencephalographic pattern: Just look at the face. Journal of Paediatrics and Child Health, 57, 963-965.

Stern, C. (2021, June 4). Woman, 36, reveals her identical TWIN is carrying her baby after volunteering to act as surrogate following her sister's lengthy battle with infertility: 'It was the natural progression.' https://www.dailymail.co. uk/femail/article-9648931/Chicago-woman-carrying-identical-twin-sistersbaby.html

Suzuki, K., \& Ando, J. (2014). Genetic and environmental structure of individual differences in hand, foot, and ear preferences: A twin study. Laterality: Asymmetries of Body, Brain and Cognition, 19, 113-128.

Tansey, J. (2021). Olympic synchronized swimming 2021: Duet medal winners, scores and results. https://bleacherreport.com/articles/2946832-olympicsynchronized-swimming-2021-duet-medal-winners-scores-and-results

Timberlake, A. T., Furey, C. G., Choi, J., Nelson-Williams, C., Loring, E., Galm, A., Kahle, K. T., Steinbacher, D. M., Larysz, D., Persing, J. A., \& Lifton, R. P. (2017). De novo mutations in inhibitors of Wnt, BMP, and Ras/ERK signaling pathways in non-syndromic midline craniosynostosis. Proceedings of the National Academy of Sciences, 114, E7341-E7347.

Tokyo 2020. (2021, June 14). Austria's triplet sisters top artistic swimming duet podium as nine nations book their places for Tokyo 2020. https://olympics. com/tokyo-2020/en/news/austria-s-triplet-sisters-top-artistic-swimmingduet-podium-as-nine-nations-book

Torrey, T. (2021). Differences between primary, secondary, tertiary, and quaternary care: Levels of care. https://www.verywellhealth.com/primarysecondary-tertiary-and-quaternary-care- 2615354

Vitarelli, A. (2021, August 2). Rare set of identical triplets born at the Hospital of the University of Pennsylvania. https://6abc.com/itentical-triplets-rarebirth-penn-medicine-babies/10925548/

Zhao, J., Wang, W., Liu, Z., Li, X., Cai, Q., \& Yu, X. (2021). Osteosarcoma in one of identical twins: Three cases report and a literature review. Orthopaedic Surgery, 13, 1443-1451.

Zheng, M., McBride, C., Ho, C. S. H., Chan, J. K. C., Choy, K. W., \& Paracchini, S. (2020). Prevalence and heritability of handedness in a Hong Kong Chinese twin and singleton sample. BMC Psychology, 8, $1-12$. 